\title{
BMJ Open Sick leave patterns as predictors of disability pension or long-term sick leave: a 6.75-year follow-up study in municipal eldercare workers
}

\author{
Christina Malmose Stapelfeldt, ${ }^{1,2}$ Claus Vinther Nielsen, ${ }^{1,2}$ Niels Trolle Andersen, ${ }^{3}$ \\ Line Krane, ${ }^{4}$ Vilhelm Borg, ${ }^{5}$ Nils Fleten, ${ }^{4}$ Chris Jensen ${ }^{2,6}$
}

To cite: Stapelfeldt CM, Nielsen CV, Andersen NT, et al. Sick leave patterns as predictors of disability pension or long-term sick leave: a 6.75-year followup study in municipal eldercare workers. BMJ Open 2014;4:e003941.

doi:10.1136/bmjopen-2013003941

- Prepublication history and additional material for this paper is available online. To view these files please visit the journal online (http://dx.doi.org/10.1136/ bmjopen-2013-003941).

Received 2 September 2013 Revised 9 December 2013 Accepted 10 January 2014

CrossMark

For numbered affiliations see end of article.

Correspondence to Dr Christina Malmose Stapelfeldt; ChristinaMalmose. Stapelfeldt@stab.rm.dk

\section{ABSTRACT}

Objectives: The aim was to study whether a workplace-registered frequent short-term sick leave spell pattern was an early indicator of future disability pension or future long-term sick leave among municipal eldercare workers.

Setting: The municipal healthcare sector in the city of Aarhus, which is the second largest city in Denmark.

Participants: All elder care employees who worked the entire year of 2004 in the municipality of Aarhus, Denmark ( $\mathrm{N}=2774)$. The employees' sick leave days during 2004 were categorised into: 0-2 and 3-17 short (1-7 days) spells, 2-13 mixed short and long (8+ days) spells and long spells only. Student workers $(n=180)$, employees who were absent due to maternal/paternal leave $(n=536)$ and employees who did not work the entire year of $2004 \quad(n=1218)$ were not included.

Primary outcome: Disability pension and long-term sick leave ( $\geq 8$ weeks) were subsequently identified in a National register. The cumulative incidence proportion as a function of follow-up weeks was estimated using the Kaplan-Meier curve. The relative cumulative incidence (RR) of experiencing events within 352 weeks was analysed in a generalised linear regression model using the pseudo values method adjusted for age, occupation, unfavourable work factors and sick leave length.

Results: A frequent short-term and a mixed sick leave pattern showed RRs of being granted a disability pension of $2.08(95 \% \mathrm{Cl} 1.00$ to 4.35$)$ and 2.61 (95\% Cl 1.33 to 5.12$)$ compared with 0-2 short spells. The risk of long-term sick leave was significantly increased for all sick leave patterns compared with 0-2 short spells. Adding sick leave length to the models attenuated all RRs and they became non-significant.

Conclusions: Sick leave length was a better indicator of future workability than spell frequency. Preventive actions should target employees engaged in homecare. The more sick leave days the greater the preventive potential seems, irrespective of spell frequency.

\section{Strengths and limitations of this study}

- Information bias was limited in this study, due to the use of workplace-registered sick leave records and the obtainment of outcome measures from a valid national register on social benefits.

- A new statistical approach (pseudo values method) in the analyses of time-to-event data was applied by which relative cumulative incidence was achieved.

- Selection and non-differential misclassification bias may have been introduced and caused underestimated results.

\section{INTRODUCTION}

Approximately $7-10 \%$ of working age inhabitants are receiving disability pension in the Nordic countries, ${ }^{1}$ and several studies seem to agree that sick leave length is an important risk factor for future disability pension. ${ }^{2-4}$

The public sector in general and the healthcare sector in particular is challenged by high sick leave rates among homecare personnel $^{56}$; next to citizens without any labour experience, this group also has the highest probability of being granted a disability pension. ${ }^{7}$ Employees in the eldercare sector also have high levels of sickness presenteeism, that is, they go to work despite their ill health. ${ }^{8}$ Commitment to the elderly and their colleagues probably partly explains this phenomenon. Exposure to an unfavourable working environment has been shown to increase the number of presenteeism episodes; and the risk of future sick leave may well increase with more presenteeism episodes. ${ }^{8}$ Studies of general work populations have shown that work environment factors are independent risk factors for being granted a disability pension. ${ }^{9-12}$ Thus, it may be of particular importance to take work 
environment factors into consideration in studies investigating the risk of being granted a disability pension among eldercare employees.

Several factors associated with disability pension were identified by Allebeck and Mastekaasa ${ }^{13}$; thus, duration of sick leave, low socioeconomic status, high age and single men were factors consistently associated with an increased risk of receiving disability pension. However, studies vary much in terms of reported sick leave length, definition of sick leave spell patterns and methods of quantifying how these factors may affect risk. Thus, longterm sick leave spells with a duration defined as more than $14,{ }^{14} 28^{15}$ and 54 days, ${ }^{16}$ respectively, increased the risk of being granted a disability pension. However, studies exploring whether short-term sick leave spells constitute a risk of being granted a disability pension differ much in their conclusions. ${ }^{2}{ }^{17}$ Frequent sick leave spells have been found to predict recurrence of a frequent sick leave spell pattern, ${ }^{18}$ and future long-term sick leave. ${ }^{17}{ }^{18}$ It is therefore relevant to study whether a pattern of frequent short-term sick leave spells may be a predictor of disability pension because such a pattern may indicate the existence of unfavourable work environment factors and may herald reduced functioning at an early stage ${ }^{19}$ A progression in frequency and duration of sick leave spells has been described as a common sick leave track preceding disability pension. ${ }^{4} 16$

Early detection of particular sick leave patterns predicting long-term sick leave or/and disability pension could facilitate employers' and healthcare professionals' initiation of preventive strategies to maintain the employees' workability. This is of special interest in the public sector in general and in the municipal eldercare sector in particular due to the high disability rates in this sector.

\section{Objective}

The aim was to study whether a workplace-registered frequent short-term sick leave spell pattern was an early indicator of future disability pension or future long-term sick leave among municipal eldercare workers.

\section{MATERIAL AND METHODS}

\section{Study design and participants}

This study was an observational follow-up study of all elder care workers who worked the entire year of 2004 in the municipality of Aarhus, Denmark ( $\mathrm{N}=2774)$. Student workers $(n=180)$, employees who were absent due to maternal/paternal leave $(n=536)$ and employees who did not work the entire year of $2004(n=1218)$ were not included. The potential follow-up period was 6.75 years. The employer's sick leave register was merged with the national register containing data on social public transfer payments (DREAM).$^{20}$

\section{Exposure variable: sick leave patterns}

Data on absence in 2004 were retrieved from the workplace records, and spells related to sick leave were included. The dates of the first and the last day of each sick leave spell were available for each individual. A sick leave spell was counted in calendar days regardless of whether all of these days were work days or not. Overlapping, consecutive or duplicate sick leave spells were merged into a single spell.

The sick leave data were categorised into patterns with short, long or a combination of short and long spells. We defined short spells as spells lasting one to seven calendar days. This definition has also been used in other studies. ${ }^{21}{ }^{22}$ Long spells were defined as spells lasting eight calendar days or more. This definition was used in a previous study conducted on the same population. ${ }^{23}$

Based on the above-mentioned measures, the sick leave patterns were defined as patterns with: $0-2$ short spells, 317 short spells, 2-13 mixed spells and 1-5 long spells.

\section{Outcome variables: disability pension and long-term sick leave}

Outcome variables were retrieved from the DREAM register. ${ }^{20} \mathrm{~A}$ person in Denmark is registered once a week with a code representing the type of social transfer payment (sick leave benefits, disability pension, etc) received that particular week.

\section{Disability pension}

Disability pension is granted if a person's capacity for work is reduced to such an extent that it makes self-support impossible, even in a flexible working arrangement. Disability pension is available to people aged $18-65 .^{24}$ While it is possible to return to work or work part time while receiving disability benefit, these options are rarely used, and disability benefit in reality means permanent exit from the labour market.

\section{Long-term sick leave}

Long-term sick leave as outcome was defined as a period of nine consecutive weeks on sick leave. In a previous study of the municipal eldercare workers, we compared DREAM-registered sickness benefits with workplace-registered long-term sick leave. ${ }^{25} \mathrm{~A}$ 9-week optimum cut-off point in the DREAM register was required to discriminate between a sick leave spell duration of 8 weeks or more defined in the workplace register. Empirical evidence indicates that long-term absentees and in particular more than 8 weeks absentees in general have a substantially increased risk of not returning to work. ${ }^{26}$ The municipal authority responsible for the sickness benefit payout is legally bound to discuss occupational and/or vocational rehabilitation with the absentees on this period. ${ }^{27}$

\section{Potential confounders}

\section{Workplace-registered variables}

Age and occupation were dichotomised into $(\leq 40 /$ $>40$ years) and (care/non-care), respectively. The care category was defined by employees engaged in the care of the elderly, and the non-care category comprised 
employees from the administration, kitchen personnel, cleaners, staff engaged in maintenance, therapists, nurses and managers. Total sick leave days in 2004 were categorised into four groups $(0 \mathrm{~s}, 1-14,15-56$ and $>57$ days) in order to study the long-term effects of being exposed to a frequent short-term sick leave spell pattern adjusted for the effect of the total sick leave length.

\section{Questionnaire-based variables}

Questionnaire data on the working environment were collected by the National Research Centre for the Working Environment (NRCWE) in the municipal eldercare sector in Aarhus from February to July in 2005. Work factors associated with self-reported high levels of sick leave among eldercare workers ${ }^{28} 29$ were selected from the survey. These were: work pace, emotional demands, demands for hiding emotions, physical workload, influence, meaning of work, commitment to the workplace, role conflict and quality of leadership. They were scored on five-point Likert scales (always, often, sometimes, seldom and never/hardly ever) and transformed into 0-100 scores, dichotomised into favourable/unfavourable according to the mean values of the different work environment scales measured in a representative sample of working Danes in 2004/2005. ${ }^{30}$ This was carried out for all scales except for physical workload which was dichotomised according to the mean value found in our data. Response categories for bullying and threats of violence were "at least once a month", "from time to time" and "never". They were dichotomised into "at least from time to time" and "never". Reference scores were not available for bullying and threats of violence.

A new variable "unfavourable work factors" was created by counting the number of individual unfavourable work factors (range 0-11).

\section{Statistical analyses}

The cumulative incidence proportion as a function of the number of follow-up weeks was estimated using the Kaplan-Meier curve. The relative cumulative incidence of being granted a disability pension or experiencing long-term sick leave within 352 weeks was analysed in a generalised linear regression model using the pseudo values method. ${ }^{31} 32$

Entry date was 1 January 2006 and the end of follow-up was week 39 in the year of 2012. The year of 2005 was used as a 'wash-out' period. ${ }^{2}{ }^{3}$ The sick leave patterns were based on sick leave spells that started or/ and ended in 2004; spells prolonged into 2005 were excluded. According to the Danish Sickness Benefit Act, sickness benefits are not reimbursed beyond 52 weeks which, in principle, is defined as the maximum duration of a sick leave spell. ${ }^{27} \mathrm{~A}$ 1-year 'wash-out' period was, therefore, sufficient to eliminate the potential confounding effects of long-term sick leave immediately before follow-up started.
The outcome variable disability pension consisted of two measures: an event indicator (yes or no) and the time allowed to code the disability benefit in the DREAM register, ${ }^{9}$ or end of follow-up/competing risks (early retirement benefit, retirement pension or death)/censored observations (emigration), whichever came first.

Likewise, the outcome variable long-term sick leave also consisted of two measures: an event indicator (yes or no) and time to nine consecutive DREAM-registered sick leave benefit weeks, ${ }^{25}$ or end of follow-up/competing risks (early retirement benefit, retirement pension, disability pension or death)/censored observations (emigration), whichever came first.

Adjustment for age, occupation, the number of unfavourable work factors and total sick leave was carried out in two steps. To study the risk of sick leave spell frequency independent of the sick leave length, total sick leave was added to the model in the second step.

A Wald test was performed to establish the overall difference in the relative cumulative incidence of being granted a disability pension or experiencing long-term sick leave between the sick leave patterns.

Owing to few events, overfitting may be present in the models analysing the risk of being granted a disability pension when adjusting for the number of unfavourable work factors and the total number of sick leave days. However, priority was given to the identicalness of the adjustment procedure in both risk populations.

The significance level was set at $p<0.05$. The results are shown as crude and adjusted relative cumulative incidences, that is, risks (RR) and corresponding 95\% CI.

STATAV.12.1 was used as statistical software.

\section{RESULTS}

\section{Descriptive statistics}

During the 'wash-out' period, $379(13.7 \%)$ of the 2774 employees experienced long-term sick leave, experienced one of the competing risks or emigrated from Denmark and did not return before 1 January 2006. This left a total of 2393 employees (two employees experienced events or competing risks within the first week) with a total time at risk of 722203 weeks until disability pension was granted $(\mathrm{n}=80)$, until competing risks $(\mathrm{n}=606)$ were experienced or until the participants were right-censored $(n=1709)$. A total of 2367 employees (28 employees experienced an event or a competing risk within the first week) with a total time at risk of 582095 weeks until long-term sick leave $(\mathrm{n}=761)$, until competing risks $(\mathrm{n}=455)$ were experienced or until the participants were right-censored $(n=1151$; figures 1 and 2).

Table 1 shows descriptive statistics of the employees stratified according to event and exposure variables. Frequent short-term sick leave spells were more prevalent among young employees than among older colleagues in both risk populations, whereas non-frequent, 
short-term and long-term sick leave spells were more common among old employees than among younger colleagues. Mixed sick leave spells were distributed equally across the age categories.

The proportion of employees having frequent shortterm and mixed sick leave patterns was higher in the care group than in the non-care group. The prevalence of long-term sick leave was the same for both occupational groups.

The majority of employees (approximately $77 \%$ ) had less than 15 sick leave days in 2004. Among the remaining $23 \%$, the majority had a mixture of short and long spells. The mean number of unfavourable work factors was similar across the spell patterns (table 1).

\section{Disability pension}

The unadjusted RR of being granted a disability pension among responders $(\mathrm{n}=1818)$ was 2.22 (95\% CI 1.15 to 4.27) for employees exposed to a frequent short-term sick leave spell pattern, 2.63 (95\% CI 1.39 to 4.95) for employees exposed to a mixed sick leave spell pattern and 3.24 (95\% CI 1.24 to 8.47 ) for employees with a non-frequent long-term sick leave spell pattern (table 2).

Adjustment by age, occupation and number of unfavourable work factors did not alter the RR much; however, the long-term sick leave spell pattern became statistically insignificant. The overall difference in RR between the sick leave patterns remained statistically significantly different from one (table 2 ).

The RR from the exposure of any of the sick leave patterns was not statistically significantly different from one when total sick leave was added to the model.

\section{Long-term sick leave}

The unadjusted RR of experiencing long-term sick leave among responders $(\mathrm{n}=1797)$ was statistically significantly increased for all sick leave patterns compared with a non-frequent short-term spell pattern (table 3). Adjustment by age, occupation and number of unfavourable work factors attenuated the RR. The overall difference between the sick leave patterns remained statistically significantly different from one $(p<0.0001)$. Being engaged in homecare ( $\mathrm{RR}=1.37 ; 95 \% \mathrm{CI} 1.15$ to 1.62) and experiencing a one-point increase in unfavourable work factors ( $\mathrm{RR}=1.03$; 95\% CI 1.01 to 1.06) independently increased the risk with respect to long-term sick leave (table 3). Further adjustment by total sick leave did not affect the risk estimates in relation to age, occupation and unfavourable work factors. The RR associated with being exposed to a frequent short-term, a mixed and a long-term sick leave spell pattern compared with a non-frequent spell pattern was attenuated and became non-significant when adjusting for total sick leave length (table 3).

\section{DISCUSSION}

Municipal eldercare workers were followed for 6.75 years via a national register containing data on disability pensions and sickness benefits to determine whether specific workplace-registered sick leave patterns in general and frequent short-term spells in particular were early predictors of being granted disability pension or/and long-term sick leave. Employees exposed to a frequent short-term spell or a mixture of short and long spells had a significantly increased RR of being granted a disability pension compared with employees with a nonfrequent short-term sick leave spell pattern.

The risk of experiencing long-term sick leave was significantly increased for all sick leave patterns compared with a non-frequent short-term sick leave pattern. Although statistically insignificant after adjustment by

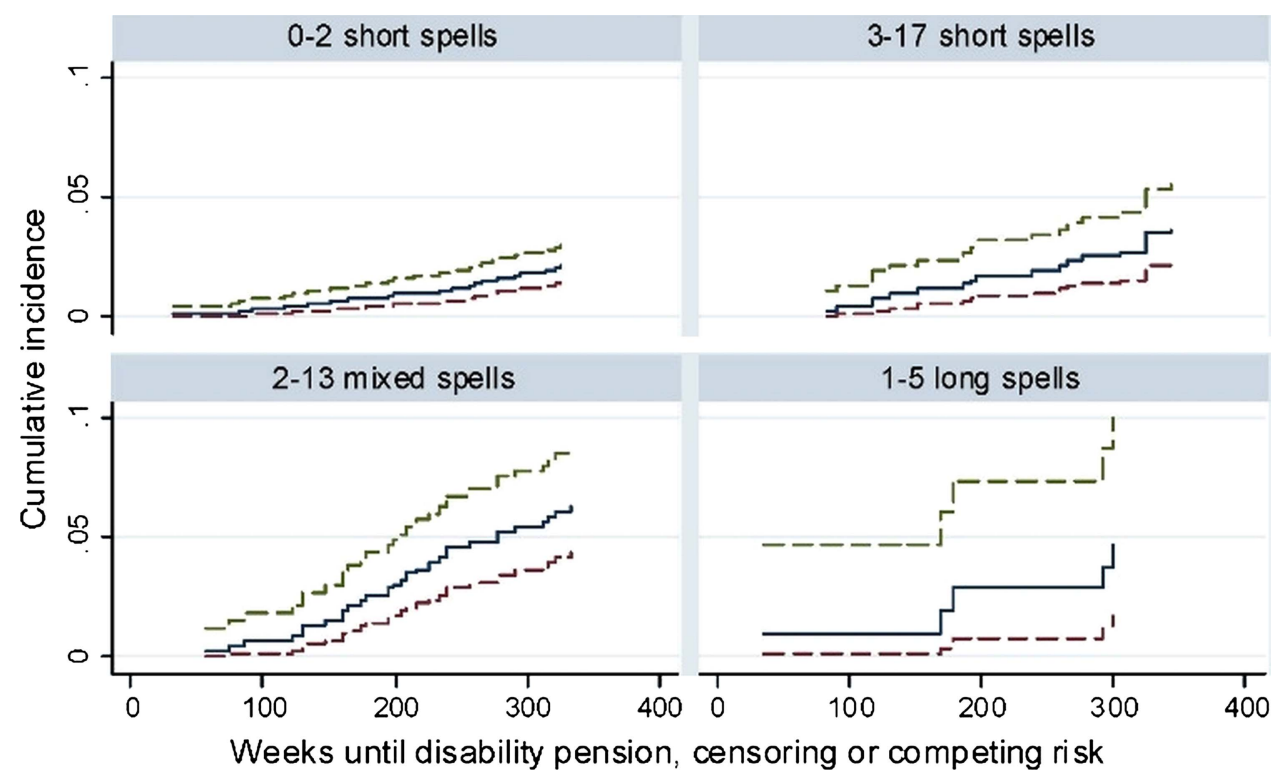

Figure 1 The cumulative incidence $(--95 \% \mathrm{Cl})$ of being granted disability pension within 352 weeks among eldercare workers, according to their sick leave pattern. 
total sick leave length, the result does not disprove that a frequent spell pattern is an indicator of future long-term sick leave, but sick leave length appears to be a better indicator of future reduced workability than spell frequency.

Previous studies have found that long-term sick leave spells lasting more than $14,{ }^{14} 28^{15}$ or 54 days ${ }^{16}$ increased the risk of being granted a disability pension. In this study, exposure to sick leave patterns defined as a frequent shortterm spell (1-7 days) or a mixture of short and long ( $>7$ days) spells increased the risk of being granted a disability pension. The prevalence of employees having less than 15 days of sick leave $(51 \%)$ and more than 14 days $(49 \%)$ was approximately the same within these two sick leave patterns. Unfortunately, we could draw no firm conclusions about the independent risk of sick leave spell frequency because adjustment by sick leave length threatened the robustness of the model due to overfitting. Hultin $e t a l^{17}$ found no increased risk of being granted a disability pension in a general population of employed persons exposed to a frequent short spell pattern defined as at least two spells lasting 1-15 days. Nor did Kivimaki et $a t^{2}$ whose increased HR of disability pensions among female municipal employees exposed to at least three short-term sick leave spells (1-3 days) became nonsignificant after adjustment for long-term spells and total sick leave. However, Wallman et $a t^{t}$ and Pedersen $e t a l,{ }^{16}$ found that sick leave patterns were commonly characterised by progression in frequency and duration of sick leave spells during 16 years before disability pension. Fortunately, disability pension is a rare event, which means that prospective risk studies need either long follow-up periods or large study populations. The follow-up periods of Hultin et $a l^{17}$ and Kivimaki et $a l^{2}$ ranged from 2 to
3.6 years; and in our study, we followed the employees for 6.75 years. These follow-up periods may be too short to detect an association between spell frequency and disability pension. Another explanation could be that spell frequency may be associated with other factors than health and that these factors may pose an independent risk for disability pension. Future studies should look into this hypothesis.

We did find an increased RR of future long-term sick leave when employees were exposed to frequent shortterm, mixed and long-term sick leave spells. This finding supports the results in studies of sick leave track records which report that such track records lead to disability pension and that frequent sick leave spell patterns pose an independent risk of future long-term sick leave. ${ }^{17} 1833$

A poor working environment has previously been found to be an independent risk factor for disability pension among general working populations, ${ }^{9-12}$ even though it should be assumed that risk is mediated by poor health. Among the eldercare workers in this study, unfavourable work factors did not increase the risk for being granted a disability pension. This result may, however, reflect underestimation of the real figures because of non-response (25\%) to the work environment questionnaire. We have previously shown that non-responders had more long-term sick leave than responders, ${ }^{25}$ and that the risk of experiencing longterm sick leave was increased among workers who were exposed to unfavourable work factors.

A poor psychosocial working environment in itself does not cause permanently reduced workability, but it may lead to health conditions that will result in a disability pension. Thus, we did not adjust for self-reported general health in the previous analyses because it may
Figure 2 The cumulative incidence (-- 95\% Cl) of experiencing long-term sick leave within 352 weeks among eldercare workers, according to their sick leave pattern.

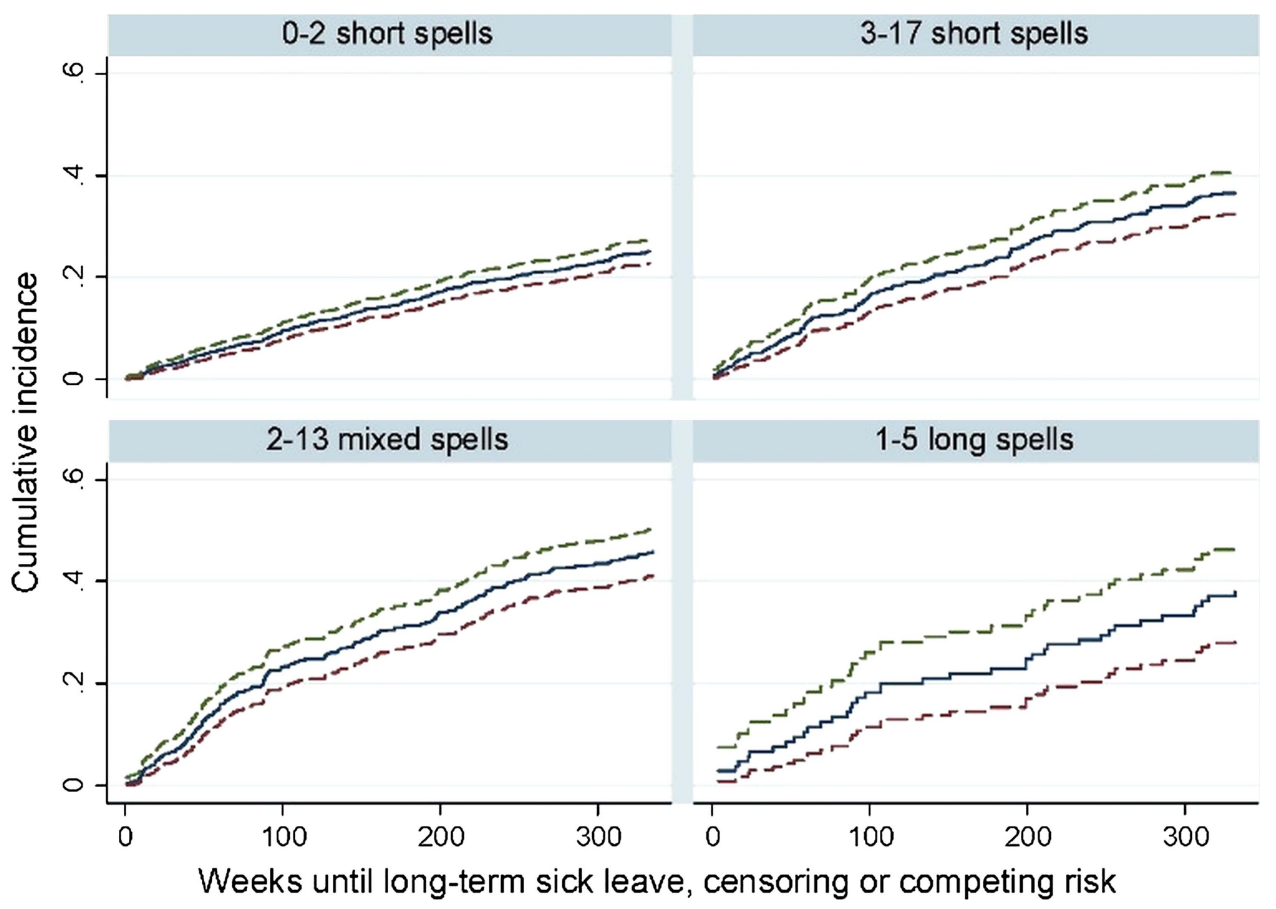


Table 1 Description of the study participants included in the risk analyses of being granted disability pension ( $\mathrm{N}=2393)$ or experiencing long-term sick leave ( $\mathrm{N}=2367$ ), respectively

\begin{tabular}{|c|c|c|c|c|c|c|c|c|c|c|c|c|c|c|c|c|}
\hline & \multicolumn{8}{|c|}{ Disability pension } & \multicolumn{8}{|c|}{ Long-term sick leave } \\
\hline & \multirow{2}{*}{\multicolumn{2}{|c|}{$\begin{array}{l}0-2 \\
\text { Short } \\
\text { spells }\end{array}$}} & \multirow{2}{*}{\multicolumn{2}{|c|}{$\begin{array}{l}-17 \\
\text { Short spells }\end{array}$}} & \multirow{2}{*}{\multicolumn{2}{|c|}{$\begin{array}{l}2-13 \\
\text { Mixed spells }\end{array}$}} & \multirow{2}{*}{\multicolumn{2}{|c|}{$\begin{array}{l}1-5 \\
\text { Long spells }\end{array}$}} & \multirow{2}{*}{\multicolumn{2}{|c|}{$\begin{array}{l}0-2 \\
\text { Short spells }\end{array}$}} & \multirow{2}{*}{\multicolumn{2}{|c|}{$\begin{array}{l}3-17 \\
\text { Short spells }\end{array}$}} & \multirow{2}{*}{\multicolumn{2}{|c|}{$\begin{array}{l}2-13 \\
\text { Mixed spells }\end{array}$}} & \multirow{2}{*}{\multicolumn{2}{|c|}{$\begin{array}{l}1-5 \\
\text { Long spells }\end{array}$}} \\
\hline & & & & & & & & & & & & & & & & \\
\hline \multicolumn{17}{|l|}{ Age in years, $n(\%)$} \\
\hline$\leq 40$ & 224 & (44) & 175 & (34) & 102 & (20) & 11 & (2) & 224 & (44) & 171 & (34) & 100 & (20) & 11 & (2) \\
\hline$>40$ & 1078 & (57) & 347 & (18) & 361 & (19) & 95 & (5) & 1071 & (58) & 342 & (18) & 354 & (19) & 94 & (5) \\
\hline \multicolumn{17}{|l|}{ Occupation, n (\%) } \\
\hline Non-care & 492 & $(66)$ & 121 & (16) & 98 & (13) & 38 & (5) & 487 & $(66)$ & 116 & (16) & 96 & (13) & 38 & (5) \\
\hline Homecare & 810 & (49) & 401 & (24) & 365 & (22) & 68 & (4) & 808 & (50) & 397 & (24) & 358 & (22) & 67 & (4) \\
\hline \multicolumn{17}{|l|}{ Categorised total sick leave days, n (\%) } \\
\hline 0 & 473 & $(100)$ & 0 & $(0)$ & 0 & $(0)$ & 0 & $(0)$ & 471 & $(100)$ & 0 & $(0)$ & 0 & $(0)$ & 0 & $(0)$ \\
\hline $1-14$ & 829 & $(60)$ & 445 & (32) & 61 & (4) & 38 & (3) & 824 & (61) & 438 & (32) & 60 & (4) & 38 & (3) \\
\hline $15-56$ & 0 & (0) & 77 & (18) & 307 & (72) & 41 & (10) & 0 & $(0)$ & 75 & (18) & 302 & (72) & 41 & (10) \\
\hline$>56$ & 0 & (0) & 0 & (0) & 95 & (78) & 27 & (22) & 0 & (0) & 0 & (0) & 92 & (78) & 26 & (22) \\
\hline $\begin{array}{l}\text { Number of unfavourable psychosocial } \\
\text { work environmental factors, mean (SD)* }\end{array}$ & 4.47 & $(2.5)$ & 4.92 & $(2.4)$ & 5.34 & $(2.5)$ & 4.94 & $(2.5)$ & 4.46 & (2.5) & 4.93 & (2.4) & 5.33 & $(2.5)$ & 4.89 & $(2.5)$ \\
\hline Event, $n(\%)$ & 27 & (2) & 19 & (4) & 29 & (6) & 5 & (5) & 325 & (25) & 188 & (37) & 208 & $(46)$ & 40 & (38) \\
\hline Competing risk, $\mathrm{n}(\%)$ & 372 & (29) & 84 & (16) & 108 & (23) & 40 & (38) & 302 & (23) & 57 & (11) & 69 & (15) & 27 & (25) \\
\hline Censoring observations, $\mathrm{n}(\%)$ & 903 & (69) & 419 & (80) & 326 & $(70)$ & 61 & (58) & 668 & (52) & 268 & (52) & 177 & (39) & 38 & (36) \\
\hline
\end{tabular}


Table 2 The RR of being granted disability pension within 352 weeks for employees exposed to a frequent short-term, mixed or long-term sick leave pattern compared with a non-frequent sick leave pattern

\begin{tabular}{|c|c|c|c|c|c|c|c|c|}
\hline \multirow[b]{3}{*}{ Pseudovalues } & \multicolumn{4}{|c|}{ Unadjusted } & \multicolumn{2}{|c|}{ Adjusted* } & \multicolumn{2}{|c|}{ Adjusted $†$} \\
\hline & \multicolumn{2}{|c|}{$\begin{array}{l}\text { Non-responders } \\
(\mathrm{n}=575)\end{array}$} & \multicolumn{2}{|c|}{$\begin{array}{l}\text { Responders } \\
(\mathrm{n}=1818)\end{array}$} & \multicolumn{4}{|c|}{ Responders ( $n=1818)$} \\
\hline & RR & $(95 \% \mathrm{Cl})$ & RR & $(95 \% \mathrm{Cl})$ & RR & $(95 \% \mathrm{Cl})$ & RR & $(95 \% \mathrm{Cl})$ \\
\hline $\begin{array}{l}\text { Overall difference between the spell } \\
\text { patterns }\end{array}$ & & $p<0.001$ & & $p=0.008$ & & $p=0.02$ & & $p=0.43$ \\
\hline \multicolumn{9}{|l|}{ Sick leave pattern } \\
\hline 0-2 short sick leave spells & 1.00 & & 1.00 & & 1.00 & & 1.00 & \\
\hline 3-17 short sick leave spells & 0.80 & (0.22 to 2.96$)$ & 2.22 & (1.15 to 4.27$)$ & 2.08 & (1.00 to 4.35$)$ & 1.76 & (0.86 to 3.62$)$ \\
\hline 2-13 mixed sick leave spells & 4.06 & (1.68 to 9.81$)$ & 2.63 & (1.39 to 4.95$)$ & 2.61 & (1.33 to 5.12$)$ & 2.04 & (0.51 to 8.10$)$ \\
\hline 1-5 long sick leave spells & 0.00 & (0.00 to 0.00$)$ & 3.24 & (1.24 to 8.47$)$ & 2.77 & (0.83 to 9.29$)$ & 1.95 & (0.29 to 13.20$)$ \\
\hline \multicolumn{9}{|c|}{ r } \\
\hline$\leq 40$ & & & & & 1.00 & & 1.00 & \\
\hline$>40$ & & & & & 1.77 & (0.57 to 5.47$)$ & 1.71 & (0.61 to 4.76$)$ \\
\hline \multicolumn{9}{|l|}{ Occupation } \\
\hline Non-care & & & & & 1.00 & & 1.00 & \\
\hline Homecare personnel & & & & & 1.29 & (0.60 to 2.78$)$ & 1.23 & (0.57 to 2.65$)$ \\
\hline Unfavourable work factor & & & & & 1.04 & (0.93 to 1.15$)$ & 1.02 & (0.90 to 1.16$)$ \\
\hline \multicolumn{9}{|l|}{ Total sick leave days } \\
\hline 0 & & & & & & & 1.00 & \\
\hline $1-14$ & & & & & & & 1.98 & (0.53 to 7.40$)$ \\
\hline $15-56$ & & & & & & & 1.74 & (0.28 to 10.82$)$ \\
\hline More than 56 & & & & & & & 3.38 & (0.50 to 22.78$)$ \\
\hline
\end{tabular}

Table 3 The RR of experiencing long-term sick leave within 352 weeks for employees exposed to a frequent short-term, mixed or long-term sick leave pattern compared with a non-frequent sick leave pattern

\begin{tabular}{|c|c|c|c|c|c|c|c|c|}
\hline \multirow[b]{3}{*}{ Pseudovalues } & \multirow{2}{*}{\multicolumn{2}{|c|}{$\begin{array}{l}\text { Unadjusted } \\
\text { Non-responders } \\
(n=570)\end{array}$}} & \multirow{2}{*}{\multicolumn{2}{|c|}{$\begin{array}{l}\text { Responders } \\
(n=1797)\end{array}$}} & \multicolumn{2}{|c|}{ Adjusted* } & \multicolumn{2}{|c|}{ Adjusted $†$} \\
\hline & & & & & \multicolumn{4}{|c|}{ Responders (n=1797) } \\
\hline & RR & $(95 \% \mathrm{Cl})$ & $\overline{\mathbf{R R}}$ & $(95 \% \mathrm{Cl})$ & $\overline{\mathbf{R R}}$ & $(95 \% \mathrm{Cl})$ & RR & $(95 \% \mathrm{Cl})$ \\
\hline Overall difference between the spell patterns & & $p<0.0001$ & & $p<0.0001$ & & $p<0.0001$ & & $p=0.19$ \\
\hline \multicolumn{9}{|l|}{ Sick leave pattern } \\
\hline 0-2 short sick leave spells & 1.00 & & 1.00 & & 1.00 & & 1.00 & \\
\hline 3-17 short sick leave spells & 1.50 & (1.13 to 1.98$)$ & 1.44 & $(1.21$ to 1.71$)$ & 1.35 & $(1.12$ to 1.62$)$ & 1.20 & (0.99 to 1.46$)$ \\
\hline 2-13 mixed sick leave spells & 2.00 & (1.53 to 2.62$)$ & 1.78 & $(1.51$ to 2.08$)$ & 1.64 & (1.40 to 1.94$)$ & 1.31 & (0.99 to 1.72$)$ \\
\hline 1-5 long sick leave spells & 1.24 & (0.68 to 2.26$)$ & 1.61 & (1.20 to 2.15$)$ & 1.52 & (1.13 to 2.03$)$ & 1.23 & (0.87 to 1.74$)$ \\
\hline \multicolumn{9}{|c|}{, } \\
\hline$\leq 40$ & & & & & 1.00 & & 1.00 & \\
\hline$>40$ & & & & & 1.04 & $(0.87$ to 1.24$)$ & 1.02 & (0.86 to 1.22$)$ \\
\hline \multicolumn{9}{|l|}{ Occupation } \\
\hline Non-care & & & & & 1.00 & & 1.00 & \\
\hline Homecare personnel & & & & & 1.37 & $(1.15$ to 1.62$)$ & 1.35 & (1.14 to 1.60$)$ \\
\hline Unfavourable work factor scores & & & & & 1.03 & (1.01 to 1.06$)$ & 1.03 & (1.00 to 1.05$)$ \\
\hline \multicolumn{9}{|l|}{ Total sick leave days } \\
\hline 0 & & & & & & & 1.00 & \\
\hline $1-14$ & & & & & & & 1.40 & (1.09 to 1.78$)$ \\
\hline $15-56$ & & & & & & & 1.52 & (1.07 to 2.15$)$ \\
\hline More than 56 & & & & & & & 1.92 & (1.29 to 2.84$)$ \\
\hline
\end{tabular}


be part of the causal pathway between sick leave pattern and disability pension. When using self-reported general health instead of sick leave patterns as the independent variable, the RR of being granted a disability pension was 4.76 (95\% CI 2.70 to 8.14 ) when exposed to a fair-to-poor self-reported general health compared with a good-to-excellent health. This corresponds with findings reported in other studies. ${ }^{3435}$

This study has several strengths. First, the exposure variable, that is, sick leave patterns, was based on a workplace register, which gave more precise sick leave measures than that of self-report. ${ }^{25}$ Sensitivity analyses of the definition of frequent short-term and mixed sick leave patterns were performed. Changing the cut-off point from 3-17 and 2-13 to 5-17 short-term sick leave spells and 5-13 mixed spells increased the relative risk (RR) of being granted a disability pension to 4.2 (95\% CI 1.0 to 17.8$)$ and 5.5 (95\% CI 0.1 to 333.7) adjusted for sick leave length, respectively. With regard to the risk of experiencing long-term sick leave, the equivalent RRs were 1.4 (95\% CI 1.0 to 1.9 ) and 1.7 (95\% CI 1.1 to 2.6). Thus, non-differential misclassification of the exposure variable may have biased the results towards the null-hypothesis.

A previous study on the association between sick leave spell patterns and work factors, ${ }^{23}$ among eldercare workers; found that of the 11 work factors, emotional demands and role conflict, were the only factors statistically significantly associated with the frequent spell patterns independently of sick leave length. Limiting the unfavourable work factor variable to include these two factors did not alter the risk estimates for either disability pension or long-term sick leave (results not shown). Misclassification of the unfavourable work factor variable seemed therefore limited.

Second, both outcomes were identified from a national register whose data are generally believed to enjoy high validity. ${ }^{25} 36$ The DREAM register has $100 \%$ coverage of granted disability pensions in Denmark. ${ }^{12}$ Long-term sick leave, defined as nine consecutive sickness benefit weeks, was validated on the same study population..$^{25}$ Misclassification of both outcome variables was therefore limited.

A third strength is the use of the pseudo values method. ${ }^{31} 32$ Even though a HR is the most common measure of association in the analysis of time-to-event data, an RR is, in general, easier to interpret than a HR. More specifically, the assumption about proportionality of hazards may not always be met; many studies do not describe whether proportionality has been checked and whether it exists in fully adjusted models or merely in the unadjusted model. This may cause results to be imprecise and may possibly lead to misleading conclusions. Finally, events exceeding a prevalence of $10 \%$ make inferences from the HR more difficult because of non-equivalence with RR.

Some limitations need to be addressed as well. Potential selection bias may have been introduced on three occasions. First, exclusion of employees on maternity/paternity leaves during 2004, which may have biased the generalisability towards women in the nonreproductive age. Second, non-response to the work environment questionnaire; this presumably gave rise to underestimated risk estimates of the exposure by mixed and long-term sick leave patterns. This presumption is supported by the smaller differences between unadjusted and adjusted results presented among responders in tables 2 and 3 than the differences we would have found if the analyses had been performed for non-responders and responders as one group.

Third, we chose a 1-year 'wash-out' period to study sick leave patterns as an early indicator of disability pension and/or long-term sick leave because we wanted to eliminate confounding due to long-term sick leave immediately before events occurred. ${ }^{23}$ However, this approach may have caused underestimation of the RR of experiencing recurrence of long-term sick leave due to selection bias; approximately $40 \%$ of those who experienced long-term sick leave during the 'wash-out' period had a mixed or a long-term sick leave pattern; the equivalent figure for those included in the final analyses was 20\% (results not shown).

We adjusted for sick leave length to get the independent risk of having a frequent sick leave pattern. This procedure may have caused overadjustment, and thus leading to bias towards the null-hypothesis in both risk estimates. Preferably we would have liked to identify a subsample of employees displaying approximately identical total sick leave lengths but having a diverse number of spells. Unfortunately, the number of individuals matching such criteria was too small.

The possible misclassification of the frequent sick leave patterns and the three types of selection bias may have caused underestimated RR in both risk estimates in general and in the RR of experiencing recurrent longterm sick leave in particular. Consequently, the results reflect rather conservative risk estimates of future reduced workability among eldercare employees. Probably, the results may be generalised to comparable female-dominated occupational settings.

\section{CONCLUSION}

Workplace-registered frequent short-term, mixed and long-term sick leave patterns were early indicators of future long-term sick leave. Sick leave length was, however, a better indicator of future reduced workability than sick leave spell frequency. Preventive actions should be targeted on employees engaged in homecare having two or more sick leave spells during a year. The longer the sick leave days were, the higher the preventive potential was, seems, irrespective of spell frequency.

\section{Author affiliations}

${ }^{1}$ Section of Social Medicine and Rehabilitation, Department of Public Health, Aarhus University, Aarhus, Denmark

${ }^{2}$ Public Health and Quality Improvement, Central Denmark Region, Aarhus, Denmark

${ }^{3}$ Section of Biostatistics, Department of Public Health, Aarhus University, Aarhus, Denmark 
${ }^{4}$ Department of Community Medicine, Faculty of Health Sciences, University of Tromsø, Tromsø, Norway

${ }^{5}$ National Research Centre for the Working Environment, Copenhagen, Denmark

${ }^{6}$ National Centre for Occupational Rehabilitation, Rauland, Norway

Contributors CMS conceived the study, carried out statistical analyses and drafted the manuscript. NTA supervised the statistical analyses. All authors participated in the design of the study, helped to draft the manuscript and interpreted the results. All authors have read, commented and approved the final version of the manuscript.

Funding This research received no specific grant from any funding agency in the public, commercial or not-for-profit sectors.

Competing interests None.

Ethics approval Approval (2012-41-1290) for using workplace-registered sick leave records, DREAM-registered sickness and disability benefit data and questionnaire data were obtained from the Danish Data Protection Agency: http://www.datatilsynet.dk/english/

Provenance and peer review Not commissioned; externally peer reviewed.

Data sharing statement No additional data are available.

Open Access This is an Open Access article distributed in accordance with the Creative Commons Attribution Non Commercial (CC BY-NC 3.0) license, which permits others to distribute, remix, adapt, build upon this work noncommercially, and license their derivative works on different terms, provided the original work is properly cited and the use is non-commercial. See: http:// creativecommons.org/licenses/by-nc/3.0/

\section{REFERENCES}

1. European Commission. Eurostat. http://appsso.eurostat.ec.europa eu/nui/show.do?dataset=spr pns ben\&lang=en

2. Kivimaki M, Forma P, Wikstrom J, et al. Sickness absence as a risk marker of future disability pension: the 10-town study. J Epidemiol Community Health 2004;58:710-11.

3. Lund T, Kivimaki M, Labriola M, et al. Using administrative sickness absence data as a marker of future disability pension: the prospective DREAM study of Danish private sector employees. Occup Environ Med 2008;65:28-31.

4. Wallman T, Wedel H, Palmer E, et al. Sick-leave track record and other potential predictors of a disability pension. A population based study of 8,218 men and women followed for 16 years. BMC Public Health 2009:9:104.

5. Barmby TA, Ercolani MG, Treble JG. Sickness absence: an international comparison. Econ J 2002;112:315-31.

6. Lund $\mathrm{T}$, Labriola M, Villadsen $\mathrm{E}$. Who is at risk for long-term sickness absence? A prospective cohort study of Danish employees. Work 2007;28:225-30.

7. CD National Social Appeals Board. Statistics about awarded anticipatory pensions. http://www.ast.dk/tal fra ankestyrelsen/

8. Elstad JI, Vabo M. Job stress, sickness absence and sickness presenteeism in Nordic elderly care. Scand J Public Health 2008;36:467-74.

9. Christensen $\mathrm{KB}$, Feveile $\mathrm{H}$, Labriola $\mathrm{M}$, et al. The impact of psychosocial work environment factors on the risk of disability pension in Denmark. Eur J Public Health 2008;18:235-7.

10. Labriola M, Feveile H, Christensen KB, et al. The impact of job satisfaction on the risk of disability pension. A 15-year prospective study. Scand J Public Health 2009;37:778-80.

11. Labriola M, Feveile $\mathrm{H}$, Christensen $\mathrm{K}$, et al. The impact of ergonomic work environment exposures on the risk of disability pension: prospective results from DWECS/DREAM. Ergonomics 2009;52:1419-22.

12. Tuchsen $\mathrm{F}$, Christensen KB, Lund $\mathrm{T}$, et al. A 15-year prospective study of shift work and disability pension. Occup Environ Med 2008;65:283-5.

13. Allebeck P, Mastekaasa A. Swedish Council on Technology Assessment in Health Care (SBU). Chapter 5. Risk factors for sick leave-general studies. Scand J Public Health Suppl 2004;63:49-108.
14. Borg K, Hensing G, Alexanderson K. Predictive factors for disability pension-an 11-year follow up of young persons on sick leave due to neck, shoulder, or back diagnoses. Scand J Public Health 2001;29:104-12.

15. Brun $\mathrm{C}$, Boggild $\mathrm{H}$, Eshoj P. [Socioeconomic risk indicators for disability pension within the Danish workforce. A registry-based cohort study of the period 1994-1998]. Ugeskr Laeger 2003;165:3315-19

16. Pedersen J, Bjorner JB, Burr $\mathrm{H}$, et al. Transitions between sickness absence, work, unemployment, and disability in Denmark 20042008. Scand J Work Environ Health 2012;38:516-26.

17. Hultin $\mathrm{H}$, Lindholm $\mathrm{C}$, Malfert $M$, et al. Short-term sick leave and future risk of sickness absence and unemployment-the impact of health status. BMC Public Health 2012;12:861.

18. Koopmans PC, Roelen CA, Groothoff JW. Risk of future sickness absence in frequent and long-term absentees. Occup Med (Lond) 2008;58:268-74.

19. Schaufeli WB, Bakker AB, Van Rhenen W. How changes in job demands and resources predict burnout, work engagement, and sickness absenteeism. J Organ Behav 2009;30:893-917.

20. The National Labour Market Authority. DREAM. Catelog 2012;12:1-38.

21. Blank N, Diderichsen F. Short-term and long-term sick-leave in Sweden: relationships with social circumstances, working conditions and gender. Scand J Soc Med 1995;23:265-72.

22. Flach PA, Krol B, Groothoff JW. Determinants of sick-leave duration: a tool for managers? Scand J Public Health 2008; 36:713-19.

23. Stapelfeldt CM, Nielsen CV, Andersen NT, et al. Are environmenta characteristics in the municipal eldercare, more closely associated with frequent short sick leave spells among employees than with total sick leave: a cross-sectional study. BMC Public Health 2013;13:578.

24. Ministry of Social Affairs and Integration. Anticipatory pension. http:// english.sm.dk/MinistryOfSocialWelfare/keystatistics/ anticipatorypension/Sider/Start.aspx

25. Stapelfeldt CM, Jensen C, Andersen NT, et al. Validation of sick leave measures: self-reported sick leave and sickness benefit data from a Danish national register compared to multiple workplace-registered sick leave spells in a Danish municipality. BMC Public Health 2012;12:661.

26. Høgelund J, Filges T, Jensen S. Long-term sickness absence-what happens and how it goes? Copenhagen: National Institute of Social Research, 2003. Report No: 03:20

27. The Ministry of Employment. The Danish Sickness Benefit Act. http://www.retsinformation.dk/Forms/r0710.aspx?id=142423

28. Borg V, Faber A, Fallentin N. Sick leave among eldercare workersa comparison between different occupations. Copenhagen, Denmark: The National Research Centre for the Working Environment, 2007. Report No.: 11

29. Borg V, Faber A, Fallentin N. Sick leave among eldercare workersa comparison between different healthcare professions. Copenhagen, Denmark: the National Research Centre for the Working Environment, 2007. Report No.: 12

30. Peitersen JH, Kristensen TS, Borg V, et al. The second version of the Copenhagen Psychosocial Questionnaire. Scand J Public Health 2010;38(Suppl 3):8-24.

31. Klein JP, Logan B, Harhoff $M$, et al. Analyzing survival curves at a fixed point in time. Stat Med 2007;26:4505-19.

32. Parner $E$, Andersen $P$. Regression analysis of censored data using the pseudo-observations. Stata J 2010;2010:408-22.

33. Koopmans PC, Roelen CAM, Groothoff JW. Frequent and long-term absence as a risk factor for work disability and job termination among employees in the private sector. Occup Environ Med 2008;65:494-9.

34. Gustafsson K, Aronsson G, Marklund S, et al. Social integration, socioeconomic conditions and type of ill health preceding disability pension in young women: a Swedish population-based study. Int $J$ Behav Med 2013. [Epub ahead of print 11 Jan 2013]

35. Halford C, Wallman T, Welin L, et al. Effects of self-rated health on sick leave, disability pension, hospital admissions and mortality. A population-based longitudinal study of nearly 15,000 observations among Swedish women and men. BMC Public Health 2012;12:1103.

36. Hjollund NH, Larsen FB, Andersen JH. Register-based follow-up of social benefits and other transfer payments: accuracy and degree of completeness in a Danish interdepartmental administrative database compared with a population-based survey. Scand J Public Health 2007;35:497-502. 